\title{
Feasibility of transradial multi-vessel percutaneous coronary intervention to both left and right coronary arteries by using Judkins left as a multipurpose guiding catheter
}

\author{
Authors: Fathima Aaysha Cader $^{\mathrm{A}}$ and Saidur Rahman Khan ${ }^{\mathrm{A}}$
}

\section{Introduction}

Appropriate guiding catheter selection in transradial percutaneous coronary intervention (PCI) is important for procedural success. Use of separate guiding catheters for multi-vessel PCI to both right and left systems requires catheter change, leading to increased radial artery spasm, patient discomfort during exchange, increased procedural time, fluoroscopy time and cost.

\section{Materials and methods}

This prospective single-centre study included patients undergoing transradial PCI of right and left systems from January 2016 to December 2018. They were assigned to two groups: single left-sided guide vs separate right and left guide. Patients requiring acute ad hoc PCI to the right coronary artery (RCA) first were excluded. We statistically analysed contrast volume, procedural time, radiation time and radial artery spasm between the two groups.

Cannulation technique: default access was right radial, unless right radial pulse was impalpable. Judkins left (JL) or appropriate left-sided catheter was engaged for left-system PCI. After completing left-system PCI, guide was disengaged, advanced to the floor of right coronary sinus, then turned clockwise while continuing to push its tip down with gentle manipulation. In some cases, a 0.035 " wire was used to assist engagement.

\section{Results and discussion}

In total 937 patients were included. Of these, 451 (48.1\%) were in the group with single left-sided guide use. In the other group, 486 (51.9\%) had separate right and left guide use. In the single guide (JL) group, 367 (81.4\%) underwent double-vessel PCI, 84 (18.6\%) underwent triple-vessel PCI. 41.5\% underwent PCI to the left anterior descending coronary artery (LAD) and RCA; $39.9 \%$ underwent PCI to the left circumflex artery (LCx) and RCA. JL 3.5 5 French (F) catheter was most frequently used

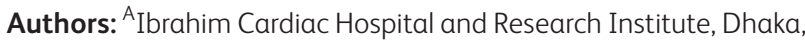
Bangladesh
(96.1\%). JL 3.56 F, JL 3.06 F and Ikari left (IL) 3.55 F guides were used in $1.5 \%, 0.9 \%$ and $1.5 \%$ of patients respectively. Compared with the group with separate guide catheters, a single guide catheter was associated with significantly lower procedural times $(\mathrm{p}=0.032)$ and radial artery spasm $(\mathrm{p}=0.02)$, most frequently seen during catheter exchange. Contrast volume was lower in the single guide catheter group. Switchover from JL to right-sided guide was $0.66 \%$, mostly in case of chronic total occlusion of RCA, necessitating better back-up support.

The search for the ideal multipurpose guide is not new, but certainly not over. Previous authors have reported the feasibility of using JL guides as multipurpose catheters, owing to their resemblance to the universal transradial diagnostic Tiger catheter, when the secondary curve of a JL catheter is straightened with a Teflon guidewire. ${ }^{1}$ The present study, however, is the first to demonstrate a significant reduction in radial spasm and procedure time with a single JL guide use. Another study reported the feasibility of 6Fr IL 3.5 as a universal guide for both-system PCI, with the limitation of a learning curve. ${ }^{2}$

A reduction in fluoroscopy and procedural times is particularly important for high-volume operators. A reduced incidence of radial spasm, increased patient comfort and reduced cost make such a universal guide particularly convenient in high-volume centres.

\section{Conclusion}

Except in certain exceptional circumstances, the use of a JL guide as a multipurpose catheter is a safe and feasible option for both-system transradial PCI, resulting in less procedural time and reduced radial spasm.

\section{Conflicts of interest}

None declared.

\section{References}

1 Kaul S, Kaul N. JL 3.5/4 as a multipurpose guiding catheter for simultaneous multivessel (left and right) transradial PCI. IHJ Cardiovasc Case Rep 2017;1:75-6.

2 Youssef AA, Hsieh Y-K, Cheng C-I, Wu J. A single transradial guiding catheter for right and left coronary angiography and intervention. EuroIntervention 2008;3:475-81. 